Artículo de revisión

\title{
Sistemas de trabajo de alto rendimiento y modelo de organización saludable frente al impacto psicológico de la COVID-19 en profesionales sanitarios
}

Rosa María Benítez-Saña*

Investigadora, Facultad de Estudios Sociales y del Trabajo, Universidad de Málaga, Málaga, España.

Responsable de Recursos Humanos, Agencia Pública Empresarial Sanitaria Costa del Sol, Málaga, España.

rosabenitez@uma.es

\section{Resumen}

El objetivo de este trabajo fue diseñar un modelo teórico de gestión de empleados en las organizaciones, cuya aplicación práctica facilite herramientas de gestión a los departamentos de recursos humanos, concretamente, en el sector sanitario. Como contribución propia, se desarrolló una metodología que aglutina diversos modelos de aplicación contrastados. Como resultado, se obtuvo un modelo teórico propio e integrador, que combina los sistemas de trabajo de alto rendimiento y el modelo de organización saludable y resiliente, como antecedentes del work engagement individual y colectivo. Se destacan posibles implicaciones prácticas y la utilidad del modelo propuesto para los departamentos de recursos humanos del sector sanitario, para hacer frente al impacto psicológico que ha tenido la pandemia por COVID-19 en los profesionales sanitarios.

Palabras clave: engagement laboral; organización saludable y resiliente; clima de compromiso; sector sanitario; COVID-19.

High-performance work systems and healthy organization model to deal with the psychological impact of COVID-19 on healthcare workers

\begin{abstract}
This work aimed to design a theoretical model of human resources management in organizations, whose practical application provides management tools for human resources departments, specifically in the health sector. As an own contribution, a methodology that brings together various contrasted application models was developed. As a result, an own theoretical and integrative model, which combines highperformance work systems and the healthy and resilient organization model, was obtained, as a background to individual and collective work engagement. The possible practical implications and the usefulness of the proposed model for human resources departments in the health sector are highlighted, to deal with the psychological impact of the COVID-19 pandemic on healthcare workers.
\end{abstract}

Keywords: work engagement; healthy and resilient organization; engagement climate; healthcare sector; COVID-19.

Sistemas de trabalho de alto desempenho e um modelo de organização saudável contra o impacto psicológico do COVID-19 nos profissionais de saúde

\section{Resumo}

O objetivo deste trabalho foi delinear um modelo teórico de gestão de colaboradores nas organizações, cuja aplicação prática forneça ferramentas de gestão aos departamentos de recursos humanos, especificamente, na área da saúde. Como contribuição própria, desenvolve-se uma metodologia que reúne vários modelos de aplicação comprovados. Como resultado, obteve-se um modelo teórico proprietário e integrador, que combina os sistemas de trabalho de alto desempenho e o modelo de organização saudável e resiliente, como antecedentes do work engagement individual e coletivo no trabalho. Destacam-se as possíveis implicações práticas e a utilidade do modelo proposto para os departamentos de recursos humanos do setor saúde, para enfrentar o impacto psicológico que a pandemia COVID-19 teve nos profissionais de saúde.

Palavras chaves: engagement no trabalho; organização saudável e resiliente; clima de compromisso; setor de saúde; COVID-19.

\footnotetext{
* Autor para dirigir correspondencia

Clasificación JEL: J28; 015; J81.
}

Cómo citar: Benítez-Saña, R. M. (2021). Sistemas de trabajo de alto rendimiento y modelo de organización saludable frente al impacto psicológico de la COVID-19 en profesionales sanitarios. Estudios Gerenciales, 37(159), 167-177. https://doi.org/10.18046/j.estger.2021.159.4376

DOI: https://doi.org/10.18046/j.estger.2021.159.4376

Recibido: 12 -oct-2020

Aceptado: 19-feb-2021

Publicado: 18-jun-2021 


\section{Introducción}

En las últimas décadas, las organizaciones han experimentado una evolución exponencial, al compás de una economía global marcada por la sucesión en cadena de importantes avances tecnológicos, la emergencia de nuevos modelos de negocio y la proliferación de los entornos de volatilidad, incertidumbre, complejidad y ambigüedad (VUCA, por sus siglas en inglés) (Bennett y Lemoine, 2014). Estos acontecimientos han sido un factor determinante en la rápida transformación de las políticas de dirección estratégica de recursos humanos (en adelante DERH).

Además, este escenario se ha visto modificado de forma repentina por diversas crisis mundiales, entre las cuales es de especial consideración la actual crisis sanitaria, ocasionada por la pandemia COVID-19, derivada del virus SARS-CoV-2 y que ha sido vaticinada como una crisis de grandes proporciones y sin precedentes desde el crack de 1929, por el impacto económico y social que supondrá a nivel global. Los datos publicados son preocupantes y ya reflejan que el impacto mundial de la pandemia ha sido mayor al esperado, en primer lugar, por la triste pérdida de vidas humanas, debido a la enfermedad, $y$, en segundo lugar, por los estragos causados tanto a nivel sanitario como económico.

Respecto a este último extremo, se han contabilizado resultados negativos alarmantes en países como Estados Unidos, con un desplome del $32,9 \%$ de su producto interno bruto (PIB); Perú, con una caída del 30,20\%; o Argentina, con una caida del $19,1 \%$ (CNN, 2020). El PIB en la eurozona sufrió un descenso medio del $12,1 \%$ y el del conjunto de la Unión Europea (UE), del $11,7 \%$ en el segundo trimestre de 2020. Esto, según Eurostat (2020), ha supuesto la mayor caída experimentada desde que comenzaron los registros de datos en 1995. En el caso de la economía española, informes recientes del Instituto Nacional de Estadística (2020) reflejan una caída del PIB del $21,5 \%$.

A la vista de los expertos, estos datos señalan que la crisis por la pandemia cambiará las empresas y la sociedad de manera extraordinaria y sin precedentes a nivel mundial. A corto plazo, la gran mayoría de las empresas para sobrevivir tendrán que buscar soluciones, más allá de sus fuentes habituales de recursos, y deberán ser capaces de anticiparse y prepararse para el impacto económico que sufrirán a causa de la crisis mundial originada por la pandemia por la COVID-19, puesto que como señalan Kissler, Tedijanto, Goldstein, Grad y Lipsitch (2020) esta pandemia puede prolongarse hasta el año 2024. Estos mismos autores señalan que es previsible que, además de la fase inicial de la actual pandemia, se sucedan nuevas olas de contagio del SARS-CoV-2 y vaticinan que en el futuro es muy probable que aparezcan más pandemias similares, lo cual condicionará de manera relevante el enfoque estratégico de la gestión de personas en las organizaciones.
Como consecuencia de estas circunstancias adversas, los trabajadores están sometidos cada vez más a mayores exigencias y demandas laborales, que dan lugar a un considerable incremento de su exposición a los riesgos psicosociales en el entorno laboral. Por tanto, las empresas, de ahora en adelante, deberán optimizar sus planes estratégicos y preparar herramientas e intervenciones para afrontar tanto los efectos físicos como psicológicos que situaciones similares producirán en sus empleados, sobre todo en determinados sectores de la actividad. En particular, en el sector sanitario, el coste psicológico que se deberá afrontar adquirirá matices muy profundos. Se requerirán sistemas de evaluación y seguimiento psicológico del personal sanitario, como consecuencia del impacto que ejercerá la pandemia por la COVID-19 sobre su salud psicológica, debido a la carga física y emocional a la que se encuentran sometidos, tal como argumentan recientes estudios (García-Iglesias et al., 2020; Santamaría, Ozamiz-Etxebarria, Rodríguez, Alboniga-Mayor y Gorrotxategi, 2020; Serrano-Ripoll et al., 2020). Sin embargo, no cabe duda de que, ante esta situación tan compleja, también se ha puesto de manifiesto la existencia de altas dosis de resiliencia en los profesionales sanitarios, quienes en los peores momentos de esta crisis sanitaria han sido capaces de hacer frente a la incertidumbre y la adversidad, y han demostrado un alto compromiso y dedicación en el desempeño profesional.

Ante este escenario, marcado por el alto impacto que la COVID-19 está ejerciendo sobre el capital humano en las organizaciones en general, y en particular en el sector sanitario, cobran especial interés todos aquellos sistemas de gestión encaminados a generar motivación y compromiso, y que están enfocados a procurar mayor bienestar y salud a los empleados. Por esta razón, y con el propósito de contribuir a la literatura, en este artículo se realiza una aproximación al concepto de work engagement (WE), tanto a nivel individual como colectivo. También se plantea como aporte una propuesta propia de modelo teórico que integra el modelo de organización saludable y resiliente (Healthy and Resilient Organization, HERO) y los sistemas de trabajo de alto rendimiento (STAR), que analiza los efectos positivos de las prácticas saludables de gestión de personas en el WE de los empleados. Como resultado, se concluye que la potenciación del WE en ambos niveles del análisis, individual y organizacionalcolectivo, contribuye a mejorar el bienestar y la salud de los empleados, así como al aumento de la ventaja competitiva y los resultados organizacionales. Finalmente, se establece una serie de implicaciones prácticas para la aplicación por parte de la DERH en las organizaciones del sector sanitario. Estas implicaciones adquieren especial interés para la DERH, considerando las previsiones sobre los efectos nocivos que tendrá la crisis de la COVID-19 en los ámbitos laboral, social y económico.

El presente trabajo se estructura de la siguiente forma: tras esta introducción, en la segunda sección 
se realiza una revisión de la literatura que aborda la conceptualización del WE, así como el modelo de demandas y recursos laborales y personales. En la tercera sección se realiza un desarrollo teórico que analiza los elementos del modelo de relaciones propuesto, se describen las consecuencias y beneficios del WE en ambos niveles de análisis y se diseña el modelo de relaciones propuesto. Finalmente, en la cuarta sección se plantean las conclusiones, las implicaciones prácticas y futuras líneas de investigación.

\section{Revisión de la literatura}

\subsection{El work engagement desde el análisis individual}

En la revista Academy of Management se publican las primeras investigaciones realizadas por Kahn (1990) sobre personal engagement y personal disengagement, dos conceptos opuestos explicados por la teoría del rol laboral como

la conexión de los miembros de una organización con sus propios roles de trabajo; en el engagement, las personas utilizan y se expresan a sí mismas física, cognitiva y emocionalmente durante el desarrollo de sus roles laborales [...] contrariamente las personas en disengagement se distancian y se autoprotegen de estos roles laborales. (Kahn, 1990, p. 694)

En su teoría, Kahn (1990) toma como base la definición que la persona hace de sí misma en relación con el trabajo que desarrolla y en qué organización lo realiza, con relación al sentimiento de orgullo de la persona por su profesión y la organización a la que pertenece.

Pese a más de dos décadas de prevalencia, el WE es un constructo relativamente novedoso, estudiado desde la corriente de la psicología positiva (Seligman y Csikszentmihalyi, 2000), que se enfoca en las fortalezas humanas y el funcionamiento óptimo del ser humano, y se centra en las experiencias positivas y la potenciación de los rasgos individuales positivos (Luthans, Avolio, Avey y Norman, 2007; Seligman y Csikszentmihalyi, 2000). Como consecuencia, en una organización capaz de enfocarse en potenciar aquellas cualidades y competencias en las que son buenas las personas que trabajan en ella y se les motiva para crecer, se genera WE y se obtienen mejores resultados.

El WE ha sido definido como "un estado mental positivo relacionado con el trabajo y caracterizado por vigor, dedicación y absorción. Más que un estado específico y momentáneo, el engagement se refiere a un estado afectivo-cognitivo que no está focalizado en un objeto, evento o situación particular." (Schaufeli, Salanova, Gonzalez-Romá y Bakker, 2002, p. 74).

Según estos autores, el vigor está caracterizado por “altos niveles de energía y resistencia mental mientras se trabaja, el deseo de esforzarse en el trabajo que se está realizando incluso a pesar de las dificultades en el camino" (Schaufeli et al., 2002, p. 74). La dedicación se puede asociar a la implicación, entusiasmo, inspiración y orgullo por el trabajo que se realiza. Finalmente, la absorción significa estar completamente enfocado y felizmente inmerso en el trabajo, unido a la sensación de que el tiempo pasa “volando" (Schaufeli et al., 2002).

En resumen, el WE consiste en la suma de conceptos como compromiso, vinculación psicológica, satisfacción personal, pasión, entusiasmo, esfuerzo y energía. La persona siente que el tiempo pasa "volando" mientras trabaja, se encuentra vinculada emocionalmente al trabajo que realiza, por lo que experimenta orgullo y entusiasmo, lo encuentra lleno de significado y supone un desafío y una fuente de inspiración, por ello se puede decir que un trabajador engaged siente pasión por su trabajo.

\subsection{El work engagement desde el análisis organizacional- colectivo}

Actualmente, son numerosos los estudios que se han centrado en el análisis del WE a nivel individual; sin embargo, desde el enfoque de DERH es interesante prestar atención al WE organizacional-colectivo, el cual puede considerarse un constructo motivacional a nivel organizacional a través de prácticas organizativas que generan recursos y capacidades dentro de la organización, con impacto en la organización en su conjunto.

El WE organizacional-colectivo se define como una percepción compartida por los miembros de una organización, aún mejor que la participación agregada a nivel individual, en la que sus miembros están colectivamente comprometidos física, cognitiva y emocionalmente con su trabajo (Barrick, Thurgood, Smith y Courtright, 2015). En esencia, se considera que si el WE existe a nivel individual en la organización, también puede existir como una construcción colectiva, y que, al igual que los individuos, los equipos de trabajo también pueden ser enérgicos y entusiastas y tener altos niveles de dedicación y absorción (Schaufeli, 2012).

De este modo, en las organizaciones en las que hay altos niveles de WE se genera el llamado clima de engagement, que hace referencia a esos sentimientos positivos compartidos en el entorno laboral; es decir, cuando un entorno laboral es desafiante y motivador, se facilita el WE, tanto individualmente como en grupo (Bakker, Albrecht y Leiter, 2011), y se generan en la organización "sentimientos compartidos de WE a nivel de equipo como ejemplo de estado anímico colectivo" (Bakker, Emmerik y Euwema, 2006, p. 466).

Estudios recientes se han ocupado de evaluar la importancia de la asociación entre los recursos centrados en la organización, el clima de compromiso de la organización y el engagement (Albrecht, Breidahl y Marty, 2018). Al crear una cultura de engagement colectivo, se fomenta el crecimiento y el desarrollo de los empleados que los motiva y estimula a prosperar, de modo que se logran resultados organizacionales inmejorables (Schaufeli, 2012). Además, se promueven aún más las espirales de compromiso debido al contagio 
de las emociones positivas en el comportamiento del grupo (Barsade, 2002). En suma, desde este punto de vista, las empresas integran los recursos existentes para crear nuevas capacidades organizativas, que luego aprovechan para crear un valor mejorado para los clientes, lo que genera mayor ventaja competitiva (Eldor, 2020) y mejores resultados organizacionales (Sirmon, Hitt, Ireland y Gilbert, 2011).

\subsection{El modelo de demandas y recursos laborales}

El modelo teórico en el que se apoya principalmente el WE, el modelo de demandas y recursos laborales (en adelante DRL) (Demerouti, Bakker, Nachreiner y Schaufeli, 2001; Bakker y Demerouti, 2007; Bakker y Demerouti, 2008; Schaufeli y Taris, 2014), propone la coexistencia de dos procesos opuestos en el entorno laboral. Por un lado, un proceso de deterioro de la salud, representado por las demandas laborales que subyacen al burnout y a la tensión laboral y, por otro, un proceso de motivación, caracterizado por aquellos recursos laborales y personales, entendidos como antecedentes de WE (Akingbola y van den Berg, 2019).

Las demandas laborales son aquellas que representan un esfuerzo físico y mental, que conllevan costes físicos y psicológicos para la persona (Demerouti et al., 2001). Destacan como principales demandas laborales a las que hacer frente a la complejidad de la tarea; la inestabilidad laboral; las demandas del desempeño; los cambios desfavorables de horarios; la sobrecarga cualitativa y cuantitativa de trabajo; los riesgos laborales; la remuneración; la responsabilidad; la ambigüedad y el conflicto de rol; la presión de la tarea, y el conflicto trabajo-casa (Schaufeli y Taris, 2014).

Los recursos laborales se definen como una serie de factores físicos, sociales u organizacionales del trabajo que facilitan ser funcionales al alcanzar los objetivos laborales, reducir los efectos negativos de las demandas laborales y los costes fisiológicos y psicológicos asociados, que estimulan el WE y favorecen el desarrollo y crecimiento personal de los empleados en las organizaciones (Demerouti et al., 2001). Autores como Schaufeli y Taris (2014) destacan recursos laborales como la autonomía, el jobcrafting (rediseño del trabajo), el desarrollo profesional, la creatividad, las recompensas económicas, el clima de innovación, el reto laboral, la participación, el conocimiento, el apoyo social de compañeros y supervisores, la cohesión/armonía de equipo, el trabajo colaborativo y la flexibilidad horaria.

\subsection{Recursos personales: el capital psicológico positivo}

Los recursos personales, incorporados posteriormente al modelo DRL por Baker y Demerouti (2008), son aquellas características positivas de las personas capaces de reducir o amortiguar el impacto negativo de las demandas, que pueden ser a la vez origen por sí mismas de procesos positivos como el crecimiento personal y profesional (Salanova y Schaufeli, 2009). En una revisión del modelo, Schaufeli y Taris (2014) destacan los principales recursos personales como competencias emocionales y mentales, motivación intrínseca, autonomía como individuo, sentido de pertenencia, sentido de competencia, organización basada en la autoestima, valores y capital psicológico positivo.

El modelo DRL tiene en cuenta el capital psicológico positivo como integrante de los recursos personales, y lo considera como un importante factor determinante de WE, que es definido como un estado de desarrollo psicológico positivo, caracterizado por cuatro componentes interrelacionados: 1) autoeficacia (confianza que tiene el empleado en las propias capacidades); 2) esperanza lvoluntad o confianza en que se lograrán los objetivos deseados);3) resiliencia (capacidad para superar circunstancias adversas); y 4) optimismo (tendencia a ver y juzgar las cosas de forma positiva) (Luthans et al., 2007; Luthans y Youssef, 2017).

En situaciones de crisis, como la originada por la pandemia de la COVID-19, la presencia de recursos personales, como el capital psicológico positivo, pueden ser cruciales para sobreponerse al impacto emocional de experiencias de ese tipo. De este modo, recursos como la autoeficacia, el optimismo y la esperanza son capaces de reforzar las capacidades y las fortalezas de las personas, y actúan como mecanismos generadores de resiliencia, tal como indican Luthans et al. (2007).

En la actualidad, el sector sanitario ha tenido que hacer frente a numerosas adversidades, debido a la pandemia por COVID-19. Los profesionales sanitarios han vivido momentos muy duros: sobrecarga de trabajo, dificultades organizativas, falta de recursos materiales, pérdida de vidas humanas, sentimiento de impotencia y sensibilidad ante el avance de la enfermedad en las personas, miedo a ser un vector de la enfermedad hacia sus familiares, temor a ser contagiados. Estas situaciones, si bien por un lado han generado emociones negativas, por otro, pueden haber servido para fortalecer el capital psicológico de los profesionales sanitarios, se podría decir que tanto a nivel individual como colectivo, debido a las experiencias vitales compartidas en circunstancias adversas.

\subsection{Aplicación práctica del modelo de demandas y recursos laborales en el sector sanitario}

La utilidad de la teoría DRL para el conocimiento de la potenciación del WE en las organizaciones ha sido corroborada por diversos estudios; por ejemplo, en el sector sanitario, por el estudio de Johansen y Sowa (2019). En el estudio llevado a cabo por Qiu et al. (2020) en un grupo de médicos de nacionalidad china, se asocia la resiliencia y el apoyo organizativo percibido con la fatiga, por lo que se llega a la conclusión de que el apoyo organizacional actúa como moderador, y es útil para aliviar la fatiga en este colectivo. El estudio de Broetje, Jenny y Bauer (2020) con enfermeras revela, por un lado, demandas laborales clave (sobrecarga de trabajo, falta 
de recompensas formales, interferencia entre trabajo y vida privadal y, por otro, los recursos laborales clave para afrontarlas lapoyo de los supervisores, gestión justa y auténtica, liderazgo transformador, relaciones interpersonales, autonomía y recursos profesionales).

El estudio de Hernández, Llorens y Rodríguez (2014) constata que las creencias de eficacia se relacionan con la calidad del servicio mediante los afectos positivos y el engagement de los profesionales sanitarios. Butucescu, Zanfirescu e Iliescu (2020), en su estudio con personal médico del sector público, utilizaron también el modelo DRL y analizaron por qué los profesionales públicos sanitarios son capaces de ir más allá de las expectativas de sus puestos.

De estos trabajos se desprende que cuanto mayor es la presencia de recursos laborales y personales adecuados en una organización, mayores niveles de WE están presentes en sus empleados y, consecuentemente, mayor capacidad tendrán estos de amortiguar el impacto negativo de las demandas laborales sobre su salud para un desempeño eficaz de sus tareas. Por tanto, es esencial para las organizaciones contar con empleados engaged con alta implicación y capacidad de gestión de los recursos personales propios.

Debido a la crisis por la COVID-19, se ha constatado esta teoría, y se ha puesto de manifiesto cómo un elevado número de profesionales sanitarios han realizado prácticamente labores de ingeniería de recursos para hacer frente a las altas e inesperadas demandas, surgidas en esta situación de alta emergencia en su sector. Estudios en el sector sanitario exponen la importancia de tener en cuenta el bienestar emocional de estos profesionales. El de Serrano-Ripoll et al. (2020), por ejemplo, destaca diversos aspectos, como la elevada frecuencia de problemas de salud mental de profesionales sanitarios al frente de emergencias causadas por epidemias virales, la influencia de factores sociodemográficos, así como el bajo nivel de evidencia científica respecto a las intervenciones para proteger la salud mental de los profesionales sanitarios.

En concreto, en estos últimos meses, el impacto de la pandemia por COVID-19 ha dado como resultado una amplia labor de gestión del conocimiento. Por parte de las organizaciones del sector sanitario, se ha intensificado la formación de los profesionales sanitarios para el uso de equipos de protección individual (EPI) y protección colectiva y se han incrementado las sesiones clínicas para el conocimiento de todos aquellos aspectos relacionados con la COVID-19. Además, se han implementado técnicas dirigidas a los profesionales sanitarios, que se pueden denominar EPI emocionales, consistentes en el conjunto de herramientas e intervenciones positivas, enfocadas a generar bienestar psicológico y ayudar a afrontar situaciones difíciles, como sesiones de mindfullness, píldoras formativas sobre alimentación saludable, pautas de cuidados de salud y bienestar físico y psíquico, prevención de la ansiedad y el estrés, ejercicios para practicar el agradecimiento y reconocimiento de la labor bien hecha, estrategias de cohesión y trabajo colaborativo para conseguir reforzar e incrementar el capital psicológico de los profesionales sanitarios.

Estas aplicaciones prácticas han conseguido amortiguar, en parte, los efectos nocivos de las altas demandas laborales que una pandemia de estas características tan extraordinarias puede ser capaz de ocasionar en los profesionales de los cuidados y la salud, cuya labor se enfoca en procurar cuidados a otras personas que lo necesitan. Esta labor, generalmente, supone un esfuerzo extraordinario para los profesionales de los cuidados, los cuales, tal y como se ha visto en estos últimos meses con la irrupción de la pandemia por el virus SARS-CoV-2, se han tenido que adaptar de manera muy rápida a situaciones adversas hasta ahora nunca vividas, marcadas por experiencias y circunstancias de alta dificultad e incertidumbre, con el elevado coste emocional y personal añadido que ha conllevado.

\section{Desarrollo teórico}

\subsection{Prácticas saludables e innovadoras de gestión de personas}

En el entorno actual en el que se desenvuelven las organizaciones, fuertemente marcado por los avances tecnológicos (digitalización, inteligencia artificial, nanotecnología, robotización de sistemas productivos, etc.), se hace necesario innovar en la gestión de personas. En torno a estos avances, se han originado numerosos cambios sociales y cambios en el trabajo y en el modelo productivo, cuya principal secuela es el alto grado de incertidumbre y las altas demandas que los empleados deben gestionar en su quehacer diario para lograr cubrir las expectativas de los clientes. Para ello, es vital contar con suficientes recursos laborales y personales, los cuales cobran un especial protagonismo. Aquellas organizaciones capaces de gestionar toda esta dinámica de manera óptima serán las que evolucionen y sobrevivan a estas vicisitudes.

Sin duda alguna, las políticas y prácticas saludables de recursos humanos han pasado a jugar un papel importante como herramienta clave para la adhesión de los empleados al proyecto de empresa, además resultan ser el motor de la implantación de estrategias organizativas para conseguir el aumento de la competitividad y la creación de valor en las organizaciones, teniendo que adoptar un rol de líder inspirador, que motive a sus colaboradores para conseguir objetivos comunes. Este importante papel se ha evidenciado en el nuevo escenario organizacional derivado de la crisis por la pandemia COVID-19. En estos últimos meses, el papel protagonista de la DERH frente a circunstancias tan adversas ha puesto de manifiesto la necesidad de que a través de ella se ejerza un liderazgo, empático e inspirador, y que oriente sus esfuerzos a conseguir que las personas que componen la organización, como su elemento clave, estén 
dispuestas a seguir a sus líderes en el emprendimiento de proyectos, se sientan comprometidas con la cultura organizacional y contribuyan con su desempeño profesional a generar valor y ventaja competitiva (Eldor, 2020) en la organización, para que alcancen altos parámetros de productividad y excelencia en el producto o servicio prestado, incluso en circunstancias adversas como las experimentadas en esta crisis.

En líneas similares, en lo que a liderazgo se refiere, se encuentra el estudio de Swensen, Gorringe, Caviness y Peters (2016) sobre el modelo de liderazgo de la Clínica Mayo, la cual diseña su sistema de gestión con el fin de desarrollar líderes culturalmente alineados, construir capital social, aumentar el engagement de los empleados, fomentar la colaboración, fomentar la colegialidad y generar confianza; esto hace confluir las funciones de la organización con el desarrollo del liderazgo y su misión. Con la aplicación de este modelo, la Clínica Mayo ha logrado generar impacto positivo en la calidad del servicio, en el paciente y en el entorno, con resultados excelentes.

Por otra parte, numerosos estudios hacen hincapié en que se pueden incrementar los niveles de WE en las organizaciones, mediante la aplicación de prácticas saludables e innovadoras en materia de gestión de personas, que ayuden a mejorar los recursos y capacidades existentes en la organización. Estas prácticas consisten en intervenciones diseñadas para incrementar los niveles de formación, cualificación, autoeficacia y bienestar y salud de los trabajadores (Acosta, Salanova y Llorens, 2011; Salanova, Llorens, Cifre y Martínez, 2012; Salanova, Llorens y Martínez, 2019; Salanova y Schaufeli, 2009). De esta forma, en las organizaciones que las aplican se generan más recursos personales y laborales, muy útiles para facilitar el afrontamiento de las demandas laborales con mayor seguridad y eficacia; con ello se consigue incrementar el WE y se obtienen resultados óptimos para ambos: organización y trabajadores.

\subsection{El modelo HERO: la organización saludable y resiliente}

El modelo HERO se destaca como modelo práctico e innovador de gestión. Las organizaciones que responden a este modelo son aquellas que, proactivamente, realizan esfuerzos sistemáticos y planificados enfocados a la mejora de la salud de sus empleados y de la organización mediante el uso de prácticas organizacionales saludables (Acosta et al., 2011; Salanova et al., 2012; Salanova et al., 2019).

El modelo HERO propone la combinación de tres componentes principales: 1) recursos y prácticas organizacionales saludables lautonomía, comunicación, soporte social, estrategias familia-trabajo, liderazgo transformacional); 2) empleados y grupos de trabajo saludables leficacia, engagement, confianza, resiliencia, afectos positivos) y 3) resultados organizacionales saludables lalto desempeño, excelencia organizacional, responsabilidad social corporativa) (Salanova et al., 2019). Además, en este modelo, las prácticas de gestión de personas se realizan en tres niveles: 1) nivel organizacional Imedidas de conciliación trabajo-familia, prácticas organizativas con enfoque en la mejora de la salud), 2) nivel de la tarea (rediseño de tareas para mejorar la autonomía, feedbackl y 3) nivel del ambiente social (liderazgo, apoyo psicosocial de supervisores y compañeros). Las prácticas organizacionales cobran un papel protagonista y son elementos clave del modelo, unidas a los recursos laborales, a los que se añade, además, la calidad organizacional (Salanova et al., 2019).

Destacan como prácticas de gestión de personas del modelo HERO aquellas que contribuyen al desarrollo de una organización saludable, tales como formación en liderazgo, feedback del desempeño, comunicación eficaz, uso de redes intranet, prevención y gestión del estrés, selección cuidadosa del personal, conciliación de la vida personal y laboral, flexibilidad laboral, evaluación del desempeño, etc. (Acosta et al., 2011; Llorens, del Líbano y Salanova, 2009). Las prácticas de gestión de personas utilizadas en el modelo HERO inciden en 10 áreas clave que fueron clasificadas por Acosta et al. (2011), tal como se muestran en la tabla 1.

Las organizaciones saludables y resilientes gozan de ambientes de trabajo que contribuyen a la productividad de la organización y mejoran la salud psicológica y física de los trabajadores (Harland, Harrison, Jones y Reiter-Palmon, 2005; Laschinger, Grau, Finegan y Wilk, 2010). Específicamente en el contexto sanitario, y también desde la perspectiva de cuidar al que cuida (Bueno y Barrientos-Trigo, 2020), se consideraría una organización sanitaria saludable y resiliente aquella que se esfuerza por garantizar la calidad de vida laboral de sus profesionales, así como por sobrevivir y prosperar en contextos de cambio económico y social, para ser capaces de crear ambientes de trabajo que contribuyan a mejorar la práctica profesional y la salud de los profesionales sanitarios; en definitiva, que su fin último sea mejorar la calidad del servicio prestado al paciente/ familiar (McAllister y Lowe, 2011; Salanova et al., 2012).

De este extremo se encargó el estudio de Hernández et al. (2014), en el que se analizó cómo las creencias de eficacia se relacionan con la calidad de servicio a través del impacto de los afectos positivos y el engagement en profesionales sanitarios.

Por ejemplo, como valor añadido, a partir de esta misma corriente de organización saludable y resiliente, nace en España la Fundación Hospital Optimista, un proyecto sin ánimo de lucro que surge para impulsar en el sector sanitario la mejora de la gestión, con un enfoque innovador, desde un planteamiento científico, empleando el modelo HERO-HOS (Healthy y Resilient Organizations Hospital), centrado en la mejora de las personas y de las organizaciones y que trabaja con la voluntad de convertir los hospitales en organizaciones sanitarias saludables y resilientes (Peláez, Salanova y Martínez, 2017). 
Tabla 1. Prácticas de recursos humanos del modelo HERO

\begin{tabular}{ll}
\hline Área de prácticas de RRHH & Acciones concretas \\
\hline 1. Recursos humanos & $\begin{array}{l}\text { Desarrollo de habilidades, desarrollo de carrera, prevención y gestión del mobbing, equidad, } \\
\text { selección cuidada de personal, retribuciones, evaluación del desempeño, formación, skilling y } \\
\text { reskilling de los profesionales. } \\
\text { Prácticas por ley, iniciativas de mejora continua en prevención, fomento de la estabilidad laboral, } \\
\text { medidas de fomento de empleo. }\end{array}$ \\
$\begin{array}{ll}\text { 2. Salud y seguridad en el trabajo } \\
\text { 3. Gestión del impacto ambiental }\end{array}$ & $\begin{array}{l}\text { Contratación de personas en riesgo de exclusión social, personas con discapacidad, víctimas de } \\
\text { violencia de género. }\end{array}$ \\
social & $\begin{array}{l}\text { Intervención de las empresas en su comunidad, responsabilidad social corporativa. } \\
\text { 5. Comunidades locales }\end{array}$ \\
6. Igualdad de oportunidades & Conciliación trabajo-familia y flexibilidad laboral, oportunidades de teletrabajo. \\
7. Comunicación e información organizacional & Comunicación eficaz a nivel ascendente, descendente y horizontal. \\
8. Códigos de conducta & Manuales y protocolos, normas y sanciones, código ético. \\
9. Relaciones interpersonales & Fomento de relaciones interpersonales en el clima laboral. \\
10. Confianza organizacional & Nivel vertical, horizontal y clientes. \\
\hline
\end{tabular}

Fuente: elaboración propia a partir de Acosta et al. (2011).

Anualmente en España, la Fundación Hospital Optimista convoca los premios Hospital Optimista, que se han convertido en un referente nacional de la humanización en el entorno sanitario, una fuerza motriz que reconoce aquellas iniciativas o prácticas positivas que contribuyen a generar un entorno más saludable para el paciente y su familia. Los premios han contado con la participación de numerosas instituciones sanitarias, las cuales desarrollan programas para la consecución de empleados positivos, un hospital optimista y pacientes satisfechos, entre ellas se encuentran el Hospital de Manises (Garrido-Lestache, 2016), el Hospital Universitario del Vinalopó, el Hospital Universitario de Torrevieja y la Agencia Pública Empresarial Sanitaria Costa del Sol y sus dos centros adscritos: el Hospital de Alta Resolución de Benalmádena, segundo premio en 2019, y la Unidad de Urgencias del Hospital Costa del Sol, finalista en 2021.

\subsection{Sistemas de trabajo de alto rendimiento}

Por otra parte, en cuanto a los sistemas para la gestión de personas, de forma innovadora, adquieren excepcional interés los sistemas de trabajo de alto rendimiento (STAR), cuyo modelo de tres dimensiones (habilidad, motivación y oportunidad) ha sido adaptado por distintos autores (Lertxundi y Landeta, 2011; Luna-Arocas y Camps-Torres, 2013; Stirpe y Revilla, 2013; Tang y Tang, 2012), en el que se incluyen intervenciones organizacionales en dichas dimensiones. La habilidad se refiere a áreas como políticas de selección (procesos exhaustivos y calidad en la selección) y formación y desarrollo (inversión en acciones formativas, planes de formación). La motivación se ocupa de las políticas retributivas y compensación (evaluación del desempeño) y de la comunicación lcanales de comunicación adecuados, buzones de sugerencias, bancos de ideas, tablones de anuncios). La oportunidad tiene que ver con el trabajo en equipo lgrupos de trabajo autónomos sistematizados para resolución de problemas, calidad, proyectos) y la participación (participación y autonomía de los empleados en las decisiones, estimulación de la creatividad y la iniciativa, las relaciones de confianza y cooperación) (Luna-Arocas y Camps-Torres, 2013; Lertxundi y Landeta, 2011).

Por su parte, Stirpe y Revilla (2013) afirman además que la utilización de este tipo de prácticas es señal de reconocimiento y compromiso por parte del empleador, y consideran que el hecho de realizar una selección cuidadosa del personal que compondrá la plantilla indica que la empresa valora a sus empleados. Además, señalan como ejemplo de prácticas basadas en STAR la participación en la toma de decisiones y en los círculos de calidad, que son interpretadas por los empleados como un modo de valorar su contribución. Al sentir que son valorados y que sus opiniones son apreciadas por sus empleadores, los trabajadores se encuentran motivados y desean corresponder a la empresa, mostrando comportamientos productivos, proactivos e innovadores y actitudes positivas hacia la tarea, lo que añade valor a su puesto de trabajo (engagement).

\subsection{Consecuencias del work engagement: resultados organizacionales saludables}

A través de los estudios analizados en este trabajo, se han descrito numerosas consecuencias positivas del WE que se traducen en ganancias mutuas, dado que, además del bienestar del trabajador, se demuestra que los sentimientos de satisfacción, orgullo, entusiasmo y fuerte vínculo emocional por su trabajo repercuten directamente en la tarea que realizan; por lo tanto, mejora la productividad y la calidad en el servicio que prestan al cliente, el cual percibe este servicio en términos de satisfacción de sus expectativas, lo cual redunda finalmente en la mejora exponencial de los resultados organizacionales. Schaufeli y Taris (2014) 
identificaron diversas consecuencias del WE que clasificaron según la siguiente descripción:

a) Consecuencias en los trabajadores: satisfacción vital, compromiso con la organización, empleabilidad, buena salud percibida, comportamiento innovador, proactividad, desempeño extra-rol, productividad, interferencia positiva trabajo-casa, felicidad laboral, etc.

b) Consecuencias en los clientes: calidad del servicio, fidelización y satisfacción del cliente.

c) Consecuencias en la organización: mejora de la productividad y resultados económicos y sociales.

d) Consecuencias en la sociedad: cabe destacar la huella social que una organización saludable añade a su entorno, dado que una organización que se preocupa por la salud y bienestar de sus empleados, por mejorar su calidad de vida laboral - con el consiguiente reflejo en su vida personal-, así como de ofrecer unos servicios y productos de excelente calidad supone un valor añadido y contribuye a una sociedad mejor. Así también lo destacan Salanova et al. (2019), quienes argumentan que las consecuencias de aplicar buenas prácticas organizacionales en términos de responsabilidad social empresarial son cuantiosas: la imagen de marca, el respeto por el medio ambiente y la justicia empresarial, por su alta contribución en la sociedad.

3.5 Modelo de relaciones: la organización saludable, positiva y resiliente

Como proposición teórica propia, en atención a la revisión de la literatura expuesta, se propone en la figura 1 un modelo de relaciones entre los conceptos analizados para su posible aplicación en organizaciones del sector sanitario, también aplicable a otros sectores.

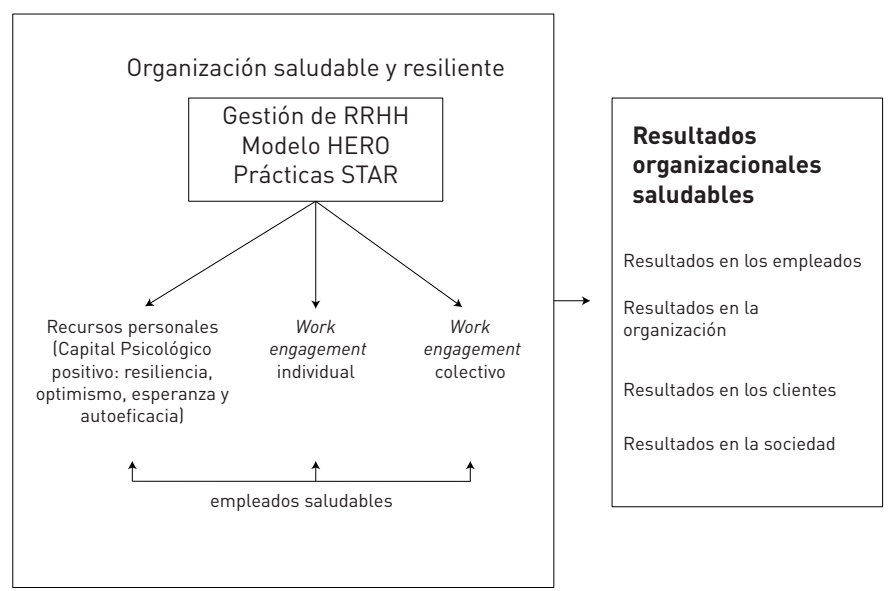

Figura 1. Modelo de organización positiva, saludable y resiliente Fuente: elaboración propia.

El modelo que aquí se propone describe la organización positiva, saludable y resiliente como aquella que integra las prácticas saludables e innovadoras de recursos humanos basadas en STAR (Lertxundi y Landeta, 2011; Luna-Arocas y Camps-Torres, 2013;
Stirpe y Revilla, 2013; Tang y Tang, 2012) y las prácticas organizacionales saludables del modelo HERO (Acosta et al., 2011; Salanova et al., 2019), como antecedentes de los recursos laborales y personales y generadoras de WE, en alineación con el modelo DRL de WE (Bakker y Demerouti, 2007, Bakker y Demerouti, 2008; Demerouti et al., 2001). De este modo, las prácticas de gestión de personas basadas en STAR y en el modelo HERO tienen relación directa con la generación de mayores recursos laborales y personales que logran potenciar el WE de los empleados, así como el WE organizacional-colectivo.

Finalmente, gracias a los altos niveles de WE en los trabajadores y en la organización, se consiguen resultados positivos en varios niveles: en los empleados, encaminados a la mejora de las condiciones laborales y la motivación, el bienestar y la salud, el desarrollo personal y profesional y el compromiso de los profesionales; en la organización, materializados en una disminución del absentismo, la accidentalidad y la rotación de la plantilla; y en los clientes, respecto a la mejora de la calidad del servicio y su satisfacción (Salanova, Agut y Peiró, 2005; Salanova y Schaufeli, 2009).

\section{Conclusiones}

En este artículo, que contribuye a la literatura con la propuesta de un modelo teórico, se analiza el WE, desde el nivel individual y también organizacional-colectivo, que pone en relación las prácticas saludables e innovadoras de gestión de personas basadas en las prácticas STAR y el modelo de organización saludable HERO, implicadas en la generación de recursos laborales y personales, lo que las caracteriza como antecedentes de WE. Cabe destacar la utilidad del modelo propuesto para la DERH en la gestión eficaz de los recursos humanos, así como para hacer frente a las demandas laborales cada vez más crecientes en el dinámico entorno económico actual. Por otra parte, se ha analizado en profundidad la filosofía de la organización positiva, saludable y resiliente que se enfoca en el bienestar del empleado, para favorecer y optimizar el WE al minimizar los efectos negativos de los riesgos psicosociales del empleado.

Con la implantación de un modelo de organización positiva, saludable y resiliente, se pueden generar beneficios en diversos niveles. En primer lugar, en el individual, dado que el empleado se siente implicado y motivado, encuentra sentido y significado al trabajo que realiza, experimenta sentido de pertenencia y bienestar, lo que tiene como consecuencia un nivel mayor de desempeño. En segundo lugar, existen ventajas a nivel organizacional y grupal, dado que un empleado engaged es capaz de contagiar de ese sentimiento de engagement a otros empleados y grupos de una misma organización, lo que desencadena un entorno o clima laboral de engagement. Con la aplicación de este modelo metodológico en las organizaciones, se consiguen no solo empleados y equipos de trabajo saludables y resilientes, sino también organizaciones positivas, saludables, 
resilientes y sostenibles, con alto impacto positivo en sus empleados, en el entorno y en la sociedad.

En cuanto a las implicaciones prácticas de los elementos analizados y que pueden ser de aplicación para la $\mathrm{DERH}$, los estudios han demostrado, de manera clara y contundente, que las organizaciones con empleados con altos niveles de WE resultan ser más competitivas, generan más valor y obtienen mejores resultados organizacionales. Con las prácticas organizacionales saludables, se contribuye a la mejora del bienestar y la salud del empleado, lo que a su vez facilita la generación de recursos laborales y personales, con los que hacer frente de manera eficaz a las complejas demandas de la actual economía, en la que, para sobrevivir, las organizaciones han de estar enfocadas en cumplir con las expectativas de los clientes en términos de calidadexcelencia. El fomento del clima de engagement por parte de la organización favorece la mejora de sus resultados, traducido en mayor satisfacción y lealtad de los clientes, mayores beneficios y mejor cuenta de resultados.

La DERH en las organizaciones actuales, para lograr alcanzar sus objetivos, superar las exigencias del mercado cambiante y sobrevivir a entornos VUCA, ha de ser altamente eficaz en su misión, por lo que debe ejercer un liderazgo transformacional e inspirador hacia sus empleados, para lograr que vayan más allá de los requerimientos y las expectativas derivadas de su puesto de trabajo, que sean proactivos, con un alto grado de autonomía en el desempeño, que tengan actitud creativa e innovadora, autoeficacia y voluntad de aprendizaje, que lleven a cabo sus tareas con energía y sean capaces de transmitir optimismo y dinamismo a los equipos de trabajo en los que se desenvuelven, con amplias habilidades sociales, interpersonales y comunicativas. En definitiva, para tener éxito, las organizaciones han de contar con empleados engaged o comprometidos con su trabajo y con altos niveles de capital psicológico.

Queda claro que con la puesta en marcha de prácticas basadas en STAR y en el modelo HERO se contribuye a mejorar el clima de engagement, lo que se consigue mejorando los sistemas de selección en los que se atraiga el talento adecuado para cada puesto de la organización, con base en planes de desarrollo profesional y formación orientados a mejorar el capital psicológico de los empleados. Estas herramientas, junto con otras diseñadas para la participación, la comunicación, el desarrollo de equipos, etc., son de gran utilidad en las organizaciones, contribuyen a la tan necesaria generación de WE y tienen además amplia repercusión en los resultados de negocio de la organización.

La crisis generalizada por la pandemia de la COVID-19 ha supuesto una debacle para muchas organizaciones, que tras numerosos esfuerzos no han aguantado su desafío, y aquellas que se han mantenido a flote han tenido que innovar y reinventarse para sobrevivir. Esto último ha puesto de manifiesto que en adelante será clave, para las organizaciones en general, incrementar la apuesta por la salud y bienestar de sus profesionales y, sobre todo, con mayor hincapié en aquellas organizaciones cuyos profesionales prestan los llamados servicios esenciales y trabajos humanos, en las que cabe resaltar nuevamente la importancia de cuidar al que cuida (Bueno y Barrientos-Trigo, 2020).

Esta evidente necesidad de saber adaptarse para sobrevivir hace necesario seguir invirtiendo en la formación y desarrollo profesional de los empleados, haciéndoles partícipes de las decisiones de la organización, fomentando la generación de ideas e innovación, prestándoles apoyo, generando confianza mutua y sentido de pertenencia, $y$, en definitiva, preocupándose por el bienestar y el buen clima laboral, que facilite a su vez un clima de engagement colectivo, lo cual se pude conseguir con la implantación de prácticas innovadoras de gestión de personas. Aquellas organizaciones que inviertan en la salud y el bienestar de sus empleados conseguirán mejores resultados en términos de rentabilidad y competitividad.

En concreto, este planteamiento es bastante válido en el caso del sector sanitario, cuya DERH ha de enfrentarse al gran reto de mejorar y mantener el bienestar y la salud de sus profesionales, para favorecer la generación de recursos con los que hacer frente a las demandas laborales, sobre todo en situaciones de crisis como la originada por la pandemia por COVID-19. Por lo tanto, el modelo propuesto en este trabajo puede incidir positivamente en este sentido y lograr organizaciones sanitarias positivas, saludables y resilientes, en las que se incremente el capital psicológico positivo y el WE, considerado a la vez una capacidad colectiva que contribuirá a generar un clima de resiliencia y de engagement favorecedor y potenciador de las fortalezas y los rasgos positivos de sus profesionales. Este modelo y su aplicación puede contribuir a la consecución de mejores resultados en la gestión de otras crisis sanitarias en el futuro.

En consecuencia, en las organizaciones sanitarias del futuro, más que nunca, serán necesarias estrategias de gestión de personas inspiradas en el modelo de prácticas organizacionales positivas saludables y resilientes, en las que se potencie el engagement individual y colectivo, así como un clima de engagement organizacional saludable para sus profesionales. Una organización que consigue mejorar la calidad de vida laboral y contar con empleados saludables y resilientes al final tendrá como resultado la calidad-excelencia de sus productos/ servicios y la satisfacción de sus clientes/usuarios, con efectos no solo en la organización, sino también en la sociedad, de manera que con estas estrategias se consiguen importantes resultados en la cifra de negocio o en la optimización de costes de una organización, y esta acaba imprimiendo una relevante huella social.

\section{Conflicto de intereses}

La autora declara no tener ningún conflicto de intereses. 


\section{Bibliografía}

Acosta, H., Salanova, M. y Llorens, S. (2011). ¿Qué prácticas organizacionales saludables son frecuentes en las empresas?: un estudio cualitativo. Fórum de Recerca, 16, 811-825.

Akingbola, K. y van den Berg, H. A. (2019). Antecedents, consequences, and context of employee engagement in nonprofit organizations. Review of Public Personnel Administration, 39(1), 46-74. https://doi.org/10.1177/0734371X16684910

Albrecht, S., Breidahl, E. y Marty, A. (2018). Organizational resources, organizational engagement climate, and employee engagement. Career Development International, 23(1), 67-85. https://doi.org/10.1108/CDI-04-2017-0064

Bakker, A. B., Albrecht, S. L. y Leiter, M. P. (2011). Work engagement: Further reflections on the state of play. European Journal of Work and Organizational Psychology, 20(1), 74-88.

https://doi.org/10.1080/1359432X.2010.546711

Bakker, A. B. y Demerouti, E. (2007). The job demands-resources model: State of the art. Journal of Managerial Psychology. 22(3), 309328. https://doi.org/10.1108/02683940710733115

Bakker, A. y Demerouti, E. (2008). Towards a model of work engagement. Career Development International, 13(3), 209-223. https://doi.org/10.1108/13620430810870476

Bakker, A. B., Emmerik, H. V. y Euwema, M. C. (2006). Crossover of burnout and engagement in work teams. Work and Occupations, 33(4), 464-489. https://doi.org/10.1177/0730888406291310

Barsade, S. G. (2002). The ripple effect: Emotional contagion and its influence on group behavior. Administrative Science Quarterly, 47(4), 644-675. https://doi.org/10.2307/3094912

Barrick, M. R., Thurgood, G. R., Smith, T. A. y Courtright, S. H. (2015). Collective organizational engagement: Linking motivational antecedents, strategic implementation, and firm performance. Academy of Management Journal, 58(1), 11-135. https://doi.org/10.5465/amj.2013.0227

Bennett, N. y Lemoine, G. J. (2014). What a difference a word makes: Understanding threats to performance in a VUCA world. Business Horizons, 57(3), 311-317. https://doi.org/10.1016/j.bushor.2014.01.001

Broetje, S., Jenny, G. J. y Bauer, G. F. (2020). The key job demands and resources of nursing staff: An integrative review of reviews. Frontiers in Psychology, 11, 1-17. https://doi.org/10.3389/fpsyg.2020.00084

Bueno, M. y Barrientos-Trigo, S. (2020). cuidar al que cuida: el impacto emocional de la epidemia de coronavirus en las enfermeras y otros profesionales de la salud. Enfermería Clínica, Supplement 1 , S35-S39. https://doi.org/10.1016/j.enfcli.2020.05.006

Butucescu, A., Zanfirescu, A. Ș. e Iliescu, D. (2020). The whys and wherefores of going the extra-mile as public health workers. Personality and Individual Differences, 153, 109646. https://doi.org/10.1016/j.paid.2019.109646

CNN (2020). Se desploman las economías de los países más afectados por la pandemia en América. Recuperado el 27 de septiembre de 2020 , de: https://n9.cl/918gu

Demerouti, E., Bakker, A. B., Nachreiner, F. y Schaufeli, W. B. (2001). The job demands-resources model of burnout. Journal of Applied Psychology, 86(3), 499-512. https://doi.org/10.1037//0021-9010.86.3.499

Eldor, L. (2020). How collective engagement creates competitive advantage for organizations: A business-level model of shared vision, competitive intensity, and service performance. Journal of Management Studies, 57(2), 177-209.

Eurostat (2020). Estimación del Producto Interior Bruto de la Zona Euro. Eurostat News Release. Recuperado el 27 de septiembre de 2020, de: https://n9.cl/jx7k

García-Iglesias, J. J., Gómez-Salgado, J., Martín-Pereira, J., FagundoRivera, J., Ayuso-Murillo, D., Martínez-Riera, J. R. y Ruiz Frutos, C. (2020). Impacto del SARS-CoV-2 (Covid-19) en la salud mental de los profesionales sanitarios: una revisión sistemática. Revista Española de Salud Pública, 94, 1-20.
Garrido-Lestache, A. (2016). Caso Hospital de Manises. En G. García y C. Soler (Drs.), Organizaciones saludables: diez casos de éxito (pp. 4958). Madrid: Thomson Reuters Aranzadi.

Harland, L., Harrison, W., Jones, J. R. y Reiter-Palmon, R. (2005). Leadership behaviors and subordinate resilience. Journal of Leadership \& Organizational Studies, 11(2), 2-14. https://doi.org/10.1177/107179190501100202

Hernández, C. I., Llorens, S. y Rodríguez, A. M. (2014). Empleados saludables y calidad de servicio en el sector sanitario. Anales de Psicología, 30(1), 247-258. http://dx.doi.org/10.6018/analesps.30.1.143631

Instituto Nacional de Estadística (2020). Contabilidad Nacional. Producto Interior Bruto. Recuperado el 27 de septiembre de 2020 , de: https://www.ine.es/prensa/pib_prensa.htm

Johansen, M. S. y Sowa, J. E. (2019). Human resource management, employee engagement, and nonprofit hospital performance. Nonprofit Management and Leadership, 29(4), 549-567. https://doi.org/10.1002/nml.21352

Kahn, W. A. (1990). Psychological conditions of personal engagement and disengagement at work. Academy of Management Journal, 33(4), 692-724. https://doi.org/10.5465/256287

Kissler, S. M., Tedijanto, C., Goldstein, E., Grad, Y. H. y Lipsitch, M. (2020). Projecting the transmission dynamics of SARS-CoV-2 through the postpandemic period. Science, 368(6493), 860-868. http://10.1126/science.abb5793

Laschinger, H. K. S., Grau, A. L., Finegan, J. y Wilk, P. (2010). New graduate nurses' experiences of bullying and burnout in hospital settings. Journal of Advanced Nursing, 66(12), 2732-2742. https://doi.org/10.1111/j.1365-2648.2010.05420.x

Lertxundi, A. L. y Landeta, J. (2011). Estrategia competitiva y sistemas de trabajo de alto rendimiento. Revista Europea de Dirección y Economía de la Empresa, 20(2), 73-86.

Llorens, S., del Líbano, M. y Salanova, M. (2009). Modelos teóricos de salud ocupacional. En M. Salanova (Ed.), Psicología de la Salud Ocupacional (pp. 63-93). Madrid: Síntesis.

Luna-Arocas, R. y Camps-Torres, J. (2013). Las prácticas de alto rendimiento en recursos humanos: el caso español. Tec Empresarial, 1(1), 26-30.

Luthans, F., Avolio, B. J., Avey, J. B. y Norman, S. M. (2007). Positive psychological capital: Measurement and relationship with performance and satisfaction. Personnel Psychology, 60(3), 541-572. https://doi.org/10.1111/j.1744-6570.2007.00083.x

Luthans, F. y Youssef, C. M. (2017). Psychological capital: An evidencebased positive approach. Annual Review of Organizational Psychology and Organizational Behavior, 4, 339-366. https://doi.org/10.1146/annurev-orgpsych-032516-113324

McAllister, M. y Lowe, J. (Eds.). (2011). The resilient nurse: empowering your practice. New York: Springer Publishing Company.

Peláez, M. J., Salanova, M. y Martinez, I. M. (2017). Hospital optimista: juntos podemos cambiar el mundo. Àgorade Salut 4, 277-286. http://dx.doi.org/10.6035/AgoraSalut.2017.4.29

Qiu, T., Yang, Y., Liu, C., Tian, F., Gu, Z., Yang, S. y Wu, H. (2020). The association between resilience, perceived organizational support and fatigue among Chinese doctors: A cross-sectional study. Journal of Affective Disorders. 265, 85-90. https://doi.org/10.1016/j.jad.2020.01.056

Serrano-Ripoll, M. J., Meneses-Echavez, J. F., Ricci-Cabello, I., FraileNavarro, D., Fiol-deRoque, M. A., Moreno, G. P. y Gonçalves-Bradley, D. (2020). Impact of viral epidemic outbreaks on mental health of healthcare workers: A rapid systematic review and meta-analysis. Journal of Affective Disorders. 277, 347-357 https://doi.org/10.1016/j.jad.2020.08.034

Salanova, M., Agut, S. y Peiró, J. M. (2005). Linking organizational resources and work engagement to employee performance and customer loyalty: The mediation of service climate. Journal of Applied Psychology, 90(6), 1217-1227. https://doi.org/10.1037/0021-9010.90.6.1217 
Salanova, M., Llorens, S., Cifre, E. y Martínez, I. M. (2012). We need a hero! Toward a validation of the healthy and resilient organization (HERO) model. Group y Organization Management, 37(6), 785-822. https://doi.org/10.1177/1059601112470405

Salanova, M. y Schaufeli, W. (2009). El engagement en el trabajo. Cuando el trabajo se convierte en pasión. Madrid: Alianza Editorial.

Salanova, M., Llorens, S. y Martínez, I. M. (2019). Organizaciones saludables: una mirada desde la psicología positiva. Madrid: Aranzadi.

Santamaría, M. D., Ozamiz-Etxebarria, N., Rodríguez, I. R., AlbonigaMayor, J. J. y Gorrotxategi, M. P. (2020). Impacto psicológico de la COVID-19 en una muestra de profesionales sanitarios españoles. Revista de Psiquiatría y Salud Mental, In Press. https://doi.org/10.1016/j.rpsm.2020.05.004

Schaufeli, W. (2012). Work engagement: What do we know and where do we go? Romanian Journal of Applied Psychology, 14(1), 3-10.

Schaufeli, W. B., Salanova, M., Gonzalez-Romá, V. y Bakker, A. B. (2002). The Measurement of engagement and burnout: A confirmative analytic approach. Journal of Happiness Studies, (3), 71-92. https://doi.org/10.1023/A:1015630930326

Schaufeli, W. B. y Taris, T. W. (2014). A critical review of the job demandsresources model: Implications for improving work and health. En G. Bauer y 0. Hämming (Eds.), Bridging Occupational, Organizational and Public Health (pp. 43-68). Dordrecht: Springer. https://doi.org/10.1007/978-94-007-5640-3_4
Seligman, M. y Csikszentmihalyi, M. (2000). Positive psychology: An introduction. American Psychologist, (55), 5-14. https://doi.org/10.1007/978-94-017-9088-8_18

Sirmon, D. G., Hitt, M. A., Ireland, R. D. y Gilbert, B. A. (2011). Resource orchestration to create competitive advantage: Breadth, depth, and life cycle effects. Journal of Management, 37(5), 1390-1412. https://doi.org/10.1177/0149206310385695

Stirpe, L. y Revilla, A. J. (2013). Efectos de la contratación temporal sobre los resultados de los sistemas de trabajo de alto rendimiento. Universia Business Review, (39), 14-31.

Swensen, S., Gorringe, G., Caviness, J. y Peters, D. (2016). Leadership by design: Intentional organization development of physician leaders. Journal of Management Development, 35(4), 549-570. https://doi.org/10.1108/JMD-08-2014-0080

Tang, T. W. y Tang, Y. Y. (2012). Promoting service-oriented organizational citizenship behaviors in hotels: The role of high-performance human resource practices and organizational social climates. International Journal of Hospitality Management, 31(3), 885-895. https://doi.org/10.1016/j.ijhm.2011.10.007 